\title{
Erratum to: Schrodinger's scat: a critical review of the currently available tiger (Panthera Tigris) and leopard (Panthera pardus) specific primers in India, and a novel leopard specific primer
}

Pranay Amruth Maroju ${ }^{\dagger}$, Sonu Yadav ${ }^{\dagger}$, Vishnupriya Kolipakam, Shweta Singh, Qamar Qureshi and Yadvendradev Jhala*

\section{Erratum}

Unfortunately, after publication of this article [1], it was noticed that the LSP Forward primer listed in Additional file 2 was incorrect. The corrected supplementary material can be found here.

\section{Additional file}

Additional file 2: Table S1. Sequence of species specific primer designed for leopards, it's annealing temperature and product size. Table S2. List of species mitochondrial sequences used in alignment for leopard specific primer design, with their accession numbers on $\mathrm{NCBI}$ database. (DOCX $78 \mathrm{~kb}$ )

Received: 24 October 2016 Accepted: 19 January 2017

Published online: 24 March 2017

\section{Reference}

1. Pranay Amruth Maroju, Sonu Yadav, Vishnupriya Kolipakam, Shweta Singh, Qamar Qureshi, Yadvendradev Jhala; Schrodinger's scat: a critical review of the currently available tiger (Panthera Tigris) and leopard (Panthera pardus) specific primers in India, and a novel leopard specific primer; BMC Genetics 2016 17:37; 10.1186/s12863-016-0344-y

* Correspondence: jhalay@wii.gov.in

${ }^{\dagger}$ Equal contributors

Wildlife Institute of India, Chandrabani, Dehradun 248001, India 